\title{
An independent constraint on the secular rate of variation of the gravitational constant from pulsating white dwarfs
}

\author{
Alejandro H. Córsico, ${ }^{a, b}$ Leandro G. Althaus, ${ }^{a, b}$ Enrique \\ García-Berro, ${ }^{c, d}$ and Alejandra D. Romero ${ }^{e}$ \\ ${ }^{a}$ Facultad de Ciencias Astronómicas y Geofísicas, Universidad Nacional de La Plata, \\ Paseo del Bosque s/n, (1900) La Plata, Argentina \\ ${ }^{b}$ Instituto de Astrofísica La Plata, CONICET-UNLP, Argentina \\ ${ }^{c}$ Departament de Física Aplicada, Universitat Politècnica de Catalunya, \\ c/Esteve Terrades, 5, 08860 Castelldefels, Spain \\ ${ }^{d}$ Institute for Space Studies of Catalonia, \\ c/Gran Capità 2-4, Edif. Nexus 104, 08034 Barcelona, Spain \\ ${ }^{e}$ Departamento de Astronomia, Universidade Federal do Rio Grande do Sul, \\ Av. Bento Goncalves 9500, Porto Alegre 91501-970, RS, Brazil \\ E-mail: acorsico@fcaglp.unlp.edu.ar
}

\begin{abstract}
A secular variation of the gravitational constant modifies the structure and evolutionary time scales of white dwarfs. Using an state-of-the-art stellar evolutionary code and an up-to-date pulsational code we compute the effects of a secularly varying $G$ on the pulsational properties of variable white dwarfs. Comparing the the theoretical results obtained taking into account the effects of a running $G$ with the observed periods and measured rates of change of the periods of two well studied pulsating white dwarfs, G117-B15A and R548, we place constraints on the rate of variation of Newton's constant. We derive an upper bound $\dot{G} / G \sim-1.8 \times 10^{-10} \mathrm{yr}^{-1}$ using the variable white dwarf G117-B15A, and $\dot{G} / G \sim-1.3 \times 10^{-10} \mathrm{yr}^{-1}$ using R548. Although these upper limits are currently less restrictive than those obtained using other techniques, they can be improved in a future measuring the rate of change of the period of massive white dwarfs.
\end{abstract}

Keywords: Stars, white dwarfs, gravity 


\section{Introduction}

The most commonly accepted theory of gravitation, namely General Relativity, relies on the assumption that the gravitational constant, $G$, is indeed an universal and genuine constant. However, the assumption that $G$ does not vary with time or location is just a hypothesis, though quite a reasonable an important one, which needs to be observationally corroborated. Actually, some modern grand-unification theories predict that the gravitational constant is a slowly varying function of low-mass dynamical scalar fields [1-3]. Thus, should these theories prove to be correct, it is foreseen that the gravitational constant would vary slowly over long timescales.

In recent years, the issue of the variation of fundamental constants has experienced a renewed interest, and several observational studies have scrutinized their hypothetical variations. The target of a sizable fraction of these studies $[2,3]$ has been to place constraints on the variation of the fine structure constant, $\alpha$, whereas other studies focused on the variation of proton-to-electron mass ratio, $\mu$. This stems from the fact that the properties of electromagnetic radiation are very sensitive to the precise values of these constants. In sharp contrast with the multitude of studies about whether (or not) there is evidence for a varying fine structure constant or a varying proton-to-electron mass ratio, only a few works have been devoted to study a hypothetical variation of the gravitational constant. Most likely, one of the reasons for this is the intrinsic experimental difficulties involved in determining the value of this constant [4] on Earth. As a matter of fact, the accuracy of this measurement is orders of magnitude lower than that of other fundamental constants, like $\alpha$ or $\mu$, making $G$ the fundamental constant with the poorest determination. Being this reason important, there are as well other problabe reasons, being the weakness of the gravitational interaction one of them. Hence, it is not surprising the large variety of methods aimed to place limits on a possible variation of $G$. Among them the most restrictive upper limits are those obtained using Lunar Laser Ranging, Big Bang nucleosynthesis and the luminosity function of white dwarfs in clusters.

Lunar Laser Ranging has provided an upper bound to the rate of variation of the gravitational constant $\dot{G} / G=(0.2 \pm 0.7) \times 10^{-12} \mathrm{yr}^{-1}[5]$, but it is purely local. At cosmological distances the tightest bound to the rate of variation of $G$ is that obtained from Big Bang nucleosynthesis. This bound turns out to be of the same order of magnitude $-0.3 \times 10^{-12} \mathrm{yr}^{-1} \lesssim \dot{G} / G \lesssim 0.4 \times 10^{-12} \mathrm{yr}^{-1}[6,7]$, but unfortunately is model-dependent. At intermediate ages the Hubble diagram of Type Ia supernovae has been used to constrain the rate of variation of $G$, yielding somewhat weaker constraints, $\dot{G} / G \lesssim 1 \times 10^{-11} \mathrm{yr}^{-1}$ at $z \sim 0.5[8,9]$.

White dwarfs can be used to set independent bounds on any hypothetical variation of $G$. Perhaps, the most important reason for this is that white dwarfs have very long evolutionary timescales [10]. Hence, even small rates of change of $G$ leave sizable imprints in their evolutionary properties. Nevertheless, there as well other reasons, which are at least as important as this one. In particular, white dwarfs represent the final state of evolution of stars with masses smaller than $\sim 10 M_{\odot}$. Consequently, most of the stars will end up their lives as a white dwarf. Moreover, the mechanical structure of the inner cores of white dwarfs is almost entirely supported by degenerate electrons. Thus, this structure is very sensitive to the precise value of $G$, and any small change in its value can become apparent. Additionally, white dwarf stars do not have nuclear energy sources. Thus, their evolution can be well understood in terms of a slow cooling process in which the gravothermal energy 
of the core is radiated through a thin, partially degenerate, insulating convective or radiative envelope. This cooling process is now very well understood for the luminosities of interest [11]. Additionally, it has been recently shown [12] that the specific rate at which white dwarfs cool is not only sensitive to its secular rate of variation, confirming previous theoretical evidence [13], but also to the specific value of $G$ at which the white dwarf was born [14].

In this paper we use white dwarfs to place an upper limit to the secular rate of change of the gravitational constant. To do this we use a direct measure of the evolutionary timescales of two pulsating white dwarfs, G117-B15A and R548. These two white dwarfs belong to the class of ZZ Ceti stars - also known as DAV pulsators. These stars are pulsating white dwarfs with hydrogen-rich envelopes. They have been monitored for very long periods of time (decades) and have good determinations of the periods and rates of period change of their main pulsational modes. In particular, G117-B15A is the benchmark member of the ZZ Ceti class, its pulsation periods being $\Pi=215.20 \mathrm{~s}, 270.46 \mathrm{~s}$ and $304.05 \mathrm{~s}$ [15]. The most recent determination of the rate of change of the period at $215 \mathrm{~s}$ - its main pulstion period - of this star is $\dot{\Pi}=(4.19 \pm 0.73) \times 10^{-15} \mathrm{~s} \mathrm{~s}^{-1}[16]$. R548 - that is, ZZ Ceti itself - is another DAV star that has been intensively studied for the last few decades. Both G117-B15A and R548 display similar pulsational properties, share periods in the intervals ranging from 213 to $215 \mathrm{~s}$ and from 272 to $274 \mathrm{~s}$, and are expected to be similar in structure. Since the discovery of R548, there have been multiple attempts to measure the drift rate of its pulsation period at $\sim 213.13 \mathrm{~s}$, but only very recently the rate of change of this period with time has been measured for the very first time. In particular, using 41 years of time-series photometry from 1970 to 2011 a value of $\dot{\Pi}=(3.3 \pm 1.1) \times 10^{-15} \mathrm{~s} \mathrm{~s}^{-1}$ has been obtained [17].

Our paper is organized as follows. In Sect. 2 we describe the asteroseismological models of the two pulsating white dwarfs used in this study. We discuss extensively the effects of a running $G$ in the periods and periods derivatives in Sect. 3, while in Sect. 4 we use these results to place constraints on a possible variation of the gravitational constant. Finally, in Sect. 6 we summarize our major findings, we discuss its significance and present our concluding remarks.

\section{Asteroseismological models for G117-B15A and R548}

Here, we briefly describe the characteristics of the asteroseismological models for G117-B15A and R548 employed in our analysis and obtained in a previous work [18]. We refer the interested reader to that paper for further details of the method. Briefly, a thorough asteroseismological analysis of G117-B15A and R548 (and 42 other bright DAV stars) was performed in [18] using a grid of fully evolutionary DA white dwarf models characterized by consistent chemical profiles for both the core and the envelope, and covering a wide range of stellar masses, thicknesses of the hydrogen envelope and effective temperatures. These models were generated with the LPCODE evolutionary code [19]. The evolutionary calculations were carried out from the ZAMS through the thermally-pulsing and mass-loss phases on the AGB, and finally to the domain of planetary nebulae and white dwarfs. These models represent a significant improvement over previous ones, that use simplified chemical profiles in the envelope and/or the core $[18,20]$. The effective temperature, the stellar mass and the mass of the $\mathrm{H}$ envelope of our DA white dwarf models vary within the ranges $14000 \gtrsim T_{\text {eff }} \gtrsim 9000 \mathrm{~K}$, $0.525 \lesssim M_{*} \lesssim 0.877 M_{\odot},-9.4 \lesssim \log \left(M_{\mathrm{H}} / M_{*}\right) \lesssim-3.6$, where the value of the upper limit of $M_{\mathrm{H}}$ is dependent on $M_{*}$ and fixed by prior evolution. For simplicity, the mass of He was kept fixed at the value predicted by the evolutionary computations for each sequence. 
Table 1. Characteristics of G117-B15A derived from spectroscopic analysis and results of the best asteroseismological model. The ranges of values in the second column have been derived by taking into account several spectroscopic analysis [21-25]. The quoted uncertainties in the asteroseismological model are the internal errors of our period-fitting procedure.

\begin{tabular}{lcc}
\hline \hline Quantity & Spectroscopy & Asteroseismology \\
\hline$T_{\text {eff }}[\mathrm{K}]$ & $11430-12500$ & $11985 \pm 200$ \\
$M_{*} / M_{\odot}$ & $0.530-0.622$ & $0.593 \pm 0.007$ \\
$\log g$ & $7.72-8.03$ & $8.00 \pm 0.09$ \\
$\log \left(R_{*} / R_{\odot}\right)$ & - & $-1.882 \pm 0.029$ \\
$\log \left(L_{*} / L_{\odot}\right)$ & - & $-2.497 \pm 0.030$ \\
$M_{\mathrm{He}} / M_{*}$ & - & $2.39 \times 10^{-2}$ \\
$M_{\mathrm{H}} / M_{*}$ & - & $(1.25 \pm 0.7) \times 10^{-6}$ \\
$X_{\mathrm{C}}, X_{\mathrm{O}}$ (center) & - & $0.28_{-0.09}^{+0.22}, 0.70_{-0.22}^{+0.09}$ \\
\hline \hline
\end{tabular}

Table 2. Same as Table 1, but for R548. The second column contains the spectroscopically determined values of $T_{\text {eff }}$ and $\log g$ [25], and the stellar mass [18].

\begin{tabular}{lcc}
\hline \hline Quantity & Spectroscopy & Asteroseismology \\
\hline$T_{\text {eff }}[\mathrm{K}]$ & $11990 \pm 200$ & $11627 \pm 390$ \\
$M_{*} / M_{\odot}$ & $0.590 \pm 0.026$ & $0.609 \pm 0.012$ \\
$\log g$ & $7.97 \pm 0.05$ & $8.03 \pm 0.05$ \\
$\log \left(R_{*} / R_{\odot}\right)$ & - & $-1.904 \pm 0.015$ \\
$\log \left(L_{*} / L_{\odot}\right)$ & - & $-2.594 \pm 0.025$ \\
$M_{\mathrm{He}} / M_{*}$ & - & $2.45 \times 10^{-2}$ \\
$M_{\mathrm{H}} / M_{*}$ & - & $(1.10 \pm 0.38) \times 10^{-6}$ \\
$X_{\mathrm{C}}, X_{\mathrm{O}}($ center $)$ & - & $0.26_{-0.09}^{+0.22}, 0.72_{-0.22}^{+0.09}$ \\
\hline \hline
\end{tabular}

In order to find an asteroseismological model for G117-B15A and R548, we searched for the models that minimize a quality function that measures the distance between theoretical $\left(\Pi^{\mathrm{t}}\right)$ and observed $\left(\Pi^{\mathrm{o}}\right)$ periods [18]. The theoretical periods were assessed by means of an up-to-date adiabatic, nonradial pulsation code [26] that solves the equations for linear nonradial stellar pulsations in the adiabatic approximation [27]. Pulsations in white dwarfs are associated to nonradial $g$ (gravity)-modes which are a subclass of spheroidal modes whose main restoring force is gravity. These modes are characterized by low oscillation frequencies (long periods) and by a displacement of the stellar fluid essentially in the horizontal direction $[27,28]$. A single best-fit model with the characteristics shown in Tables 1 and 2 was found for each star. The second column of these tables displays the effective temperature, gravity and stellar masss ( $T_{\text {eff }}, \log g$ and $M_{*}$ ) of G117-B15A and R548, respectively, according to spectroscopic studies. The parameters characterizing the asteroseismological models are shown in column 3. It is worth noting the very good agreement between the spectroscopically inferred values of $T_{\text {eff }}, \log g$ and $M_{*}$ and the seismological results. These asteroseismological models have proven be essential to put constraints on the mass of the axion [29, 30], so it is natural to use them to place constraints on a secularly varying $G$.

In Tables 3 and 4 we compare the observed and theoretical periods and rates of change of the period. Notably, the models reproduce very accurately the observed periods. This guarantees us that seismological models are an excellent representation of the internal structure of 
Table 3. The observed and theoretical periods of G117-B15A, along with the corresponding mode identification, and the subsequent rates of change of period with time. The theoretical ones were computed assuming $\dot{G}=0$.

\begin{tabular}{cccccc}
\hline \hline $\begin{array}{c}\Pi^{\mathrm{o}} \\
{[\mathrm{s}]}\end{array}$ & $\begin{array}{c}\Pi^{\mathrm{t}} \\
{[\mathrm{s}]}\end{array}$ & $\ell$ & $k$ & $\begin{array}{c}\dot{\Pi}^{\mathrm{o}} \\
{\left[10^{-15} \mathrm{~s} / \mathrm{s}\right]}\end{array}$ & $\begin{array}{c}\dot{\Pi}^{\mathrm{t}} \\
{\left[10^{-15} \mathrm{~s} / \mathrm{s}\right]}\end{array}$ \\
\hline- & 189.19 & 1 & 1 & - & 3.01 \\
215.20 & 215.22 & 1 & 2 & $4.19 \pm 0.53$ & 1.25 \\
270.46 & 273.44 & 1 & 3 & - & 4.43 \\
304.05 & 301.85 & 1 & 4 & - & 4.31 \\
\hline \hline
\end{tabular}

Table 4. Same as Table 3, but for R548.

\begin{tabular}{cccccc}
\hline \hline $\begin{array}{c}\Pi^{\mathrm{o}} \\
{[\mathrm{s}]}\end{array}$ & $\begin{array}{c}\Pi^{\mathrm{t}} \\
{[\mathrm{s}]}\end{array}$ & $\ell$ & $k$ & $\begin{array}{c}\dot{\Pi}^{\mathrm{o}} \\
{\left[10^{-15} \mathrm{~s} / \mathrm{s}\right]}\end{array}$ & $\begin{array}{c}\dot{\Pi}^{\mathrm{t}} \\
{\left[10^{-15} \mathrm{~s} / \mathrm{s}\right]}\end{array}$ \\
\hline 186.86 & 187.59 & 1 & 1 & - & 2.51 \\
212.95 & 213.40 & 1 & 2 & $3.3 \pm 1.1$ & 1.08 \\
274.52 & 272.26 & 1 & 3 & - & 3.76 \\
318.08 & 311.36 & 2 & 8 & - & 6.32 \\
333.64 & 336.50 & 2 & 9 & - & 8.80 \\
\hline \hline
\end{tabular}

the real stars. However, the most important fact shown in Tables 3 and 4 is that the observed rates of change of the $215 \mathrm{~s}$ period of G117-B15A and the $213 \mathrm{~s}$ period of R548 are more than 3 times larger than the theoretically expected values. This is because the $\ell=1, k=2$ mode is a mode trapped in the outer hydrogen envelope in our asteroseismological models. Mode trapping reduces the rate of change of period with time by up to a factor of $\sim 3$ if the mode is trapped in the outer hydrogen envelope, because gravitational contraction - that is still appreciable in these regions — reduces the increase in period due to cooling [31]. Since the $\ell=1, k=2$ mode is somewhat affected by gravitational contraction, it is less sensitive to cooling. However, the change of the period due to the increasing degeneracy resulting from cooling is still larger than the change due to residual contraction, and so, $\dot{\Pi}>0$. As a result, the period of the $\ell=1, k=2$ mode is still sensitive to cooling, and therefore, its rate of change reflects the evolutionary timescale of the stars. Therefore, the disagreement between the observed and theoretical values of $\dot{\Pi}$ would be a hint that G117-B15A and R548 could be cooling faster than that predicted by the standard theory of white dwarf evolution. This possible anomalous rate of cooling has been explained in terms of an additional cooling produced by axions [32-34].

\section{The rates of period change with a varying $G$}

In the previous section we have presented the periods and rates of period change of G117-B15A and R548 that do not take into account a possible secular rate of variation of the gravitational constant $G$ - see Tables 3 and 4 . We have found that the theoretically expected rate of period change of the modes with $k=2$ is markedly smaller than the value measured for G117-B15A and R548, suggesting the existence of some additional cooling mechanism in these stars. In this section we shall assume that this additional cooling is entirely due to a varying $G$. In this regard we point out that the upper bounds derived here are conservative, 


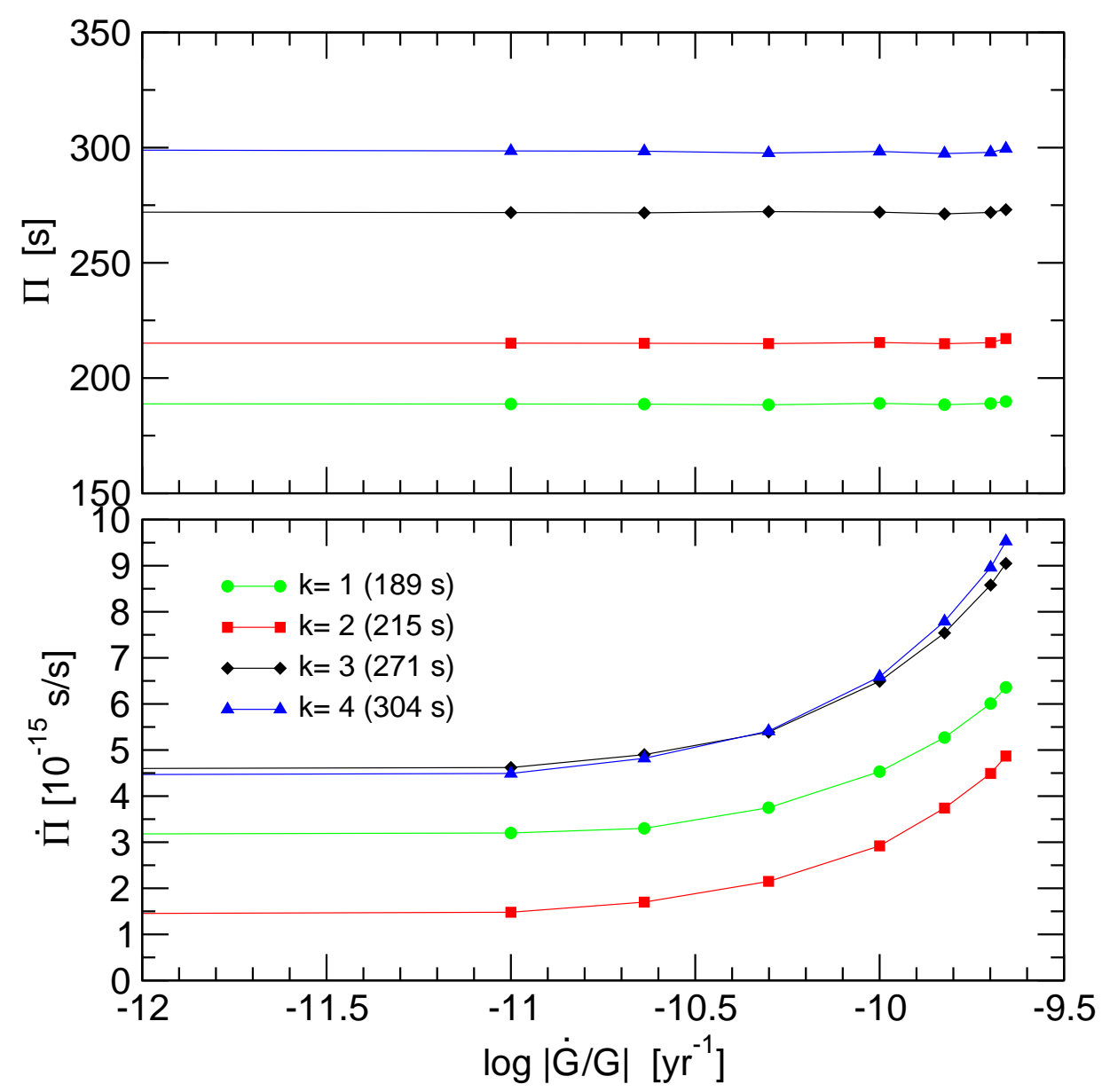

Figure 1. Upper panel: periods of the several modes of G117-B15A as a function of the value of $|\dot{G} / G|$ with $\dot{G}<0$. Lower panel: period derivatives of the same modes as a function of the secular rate of change of $G$. The effective temperature of the models is $T_{\text {eff }} \sim 11990 \mathrm{~K}$. See text for details.

since we attribute the entire discrepancy between the observed and the theoretical cooling rates of these white dwarfs - see Sect. 5 - to a varying $G$, which is an extreme case. We will moreover assume that $\dot{G}<0$, unless otherwise stated.

Following Ref. [12], we have computed a set of DA white dwarf cooling sequences considering a secularly varying $G$. In the interest of simplicity we have assumed that $\dot{G} / G$ remains constant with time. We have adopted different values for $\dot{G} / G$ and the same structural parameters $\left(M_{*}, M_{\mathrm{H}}\right)$ previously obtained in our asteroseismological models. We have considered a range of values for the rate of change of the gravitational constant $5 \times 10^{-12} \mathrm{yr}^{-1} \leq|\dot{G} / G| \leq 2.5 \times 10^{-10} \mathrm{yr}^{-1}$. It is worth emphasizing that the evolution with a varying $G$ is strongly dependent on the initial value of $G$ at the beginning of the cooling phase [12]. Accordingly, for each value of $\dot{G} / G$ we computed several sequences with different values of $G_{\mathrm{i}} / G_{0}$, where $G_{\mathrm{i}}$ stands for the value of $G$ at time $t_{\mathrm{i}}$, corresponding to the beginning of the cooling track at high effective temperature, and $G_{0}$ corresponds to the present value of $G$. Specifically, we have assumed that the variation of $G$ is exponential, and follows a simple exponential law of the form: 


$$
G(t)=G_{i} \exp \left[\frac{\dot{G}}{G}\left(t-t_{i}\right)\right],
$$

To obtain starting white dwarf configurations with the appropriate value of $G$, we simply changed $G_{0}$ by $G_{\mathrm{i}}$ at the beginning of the cooling phase (at time $t_{\mathrm{i}}$ ) and calculated the resulting structure. After a brief transitory stage, we obtain the initial configurations for our sequences. This artificial procedure to obtain the initial white dwarf configurations is justified since the subsequent evolution does not depend on the way the initial white dwarf structures are obtained. Note that the election of the values of $G_{\mathrm{i}}$ was made in such a way that $G(t) \equiv G_{0}$ at the effective temperature of these stars according to their asteroseismological models, that is, $T_{\text {eff }} \sim 11990 \mathrm{~K}$ in the case of G117-15A, and $T_{\text {eff }} \sim 11630 \mathrm{~K}$ for R548. In this way, we consistently assure that the varying value of $G$ in our evolutionary computations is coincident at the present time with the present value of the gravitational constant.

The pulsation periods for the modes with $\ell=1$ and $k=1,2,3$ and 4 of the asteroseismological model of G117-B15A for increasing values of $|\dot{G} / G|$ are depicted in the upper panel of Fig. 1. The variation of the periods is negligible, in spite of the rather wide range of values of $|\dot{G} / G|$ considered here - spanning about two orders of magnitude. This result implies that due to a varying $G$ with $\dot{G}<0$ the structure of the asteroseismological model itself is negligibly affected, in such a way that, for a fixed value of the effective temperature, the pulsation periods are largely independent of the adopted value of $|\dot{G} / G|$.

In the lower panel of Fig. 1 we display the rates of period change for the same modes. At odds with what happens with the pulsation periods, the values of the rates of period change are markedly affected by a varying $G$, substantially increasing for increasing values of $|\dot{G} / G|$. This is because that, for a decreasing value of $G$ with time, the white dwarf cooling process accelerates $[12,13]$, and this is translated into a larger secular change of the pulsation periods as compared with the situation in which $G$ is constant. In particular, the rate of period change of the mode with $k=2$, which is the relevant one in the present analysis, increases by a factor of about 5 in the range of $|\dot{G} / G|$ considered in the case $\dot{G}<0$.

For sake of conciseness, we have shown results corresponding to G117-B15A only, but the same analysis can be done for R548 and identical results are obtained. Finally, we mention that we have also computed a set of models in which we vary $G$ but we adopt $\dot{G}>0$. In this case, we found that the pulsation periods do not change, and that the rates of period change decrease for increasing values of $|\dot{G} / G|$. This case is not of direct interest in the context of this paper, because to reconcile the the difference between the theoretical and the measured values of the rates of period change in the ZZ Ceti stars G117-B15A and R548 a physical mechanism able to increase these rates must be sought. Thus, we will not consider the case of a varying $G$ with $\dot{G}>0$ in the remainder of the paper.

\section{An upper limit to the rate of variation of $G$}

Here, we focus on the mode with $k=2$, for which we have a measurement of its rate of period change for both G117-B15A and R548. In Fig. 2 we display the theoretical value of $\dot{\Pi}$ corresponding to the period $\Pi=215$ s of G117-B15A for increasing values of $|\dot{G} / G|$ (red solid curve). The dashed curves embracing the solid curve represent the uncertainty in the theoretical value of $\dot{\Pi}, \epsilon_{\dot{\Pi}}=0.09 \times 10^{-15} \mathrm{~s} \mathrm{~s}^{-1}$. This value has been obtained considering the uncertainty introduced by our lack of precise knowledge of the ${ }^{12} \mathrm{C}(\alpha, \gamma){ }^{16} \mathrm{O}$ reaction rate $-\varepsilon_{1} \sim 0.03 \times 10^{-15} \mathrm{~s} / \mathrm{s}$, and that due to the errors in the asteroseismological model - 


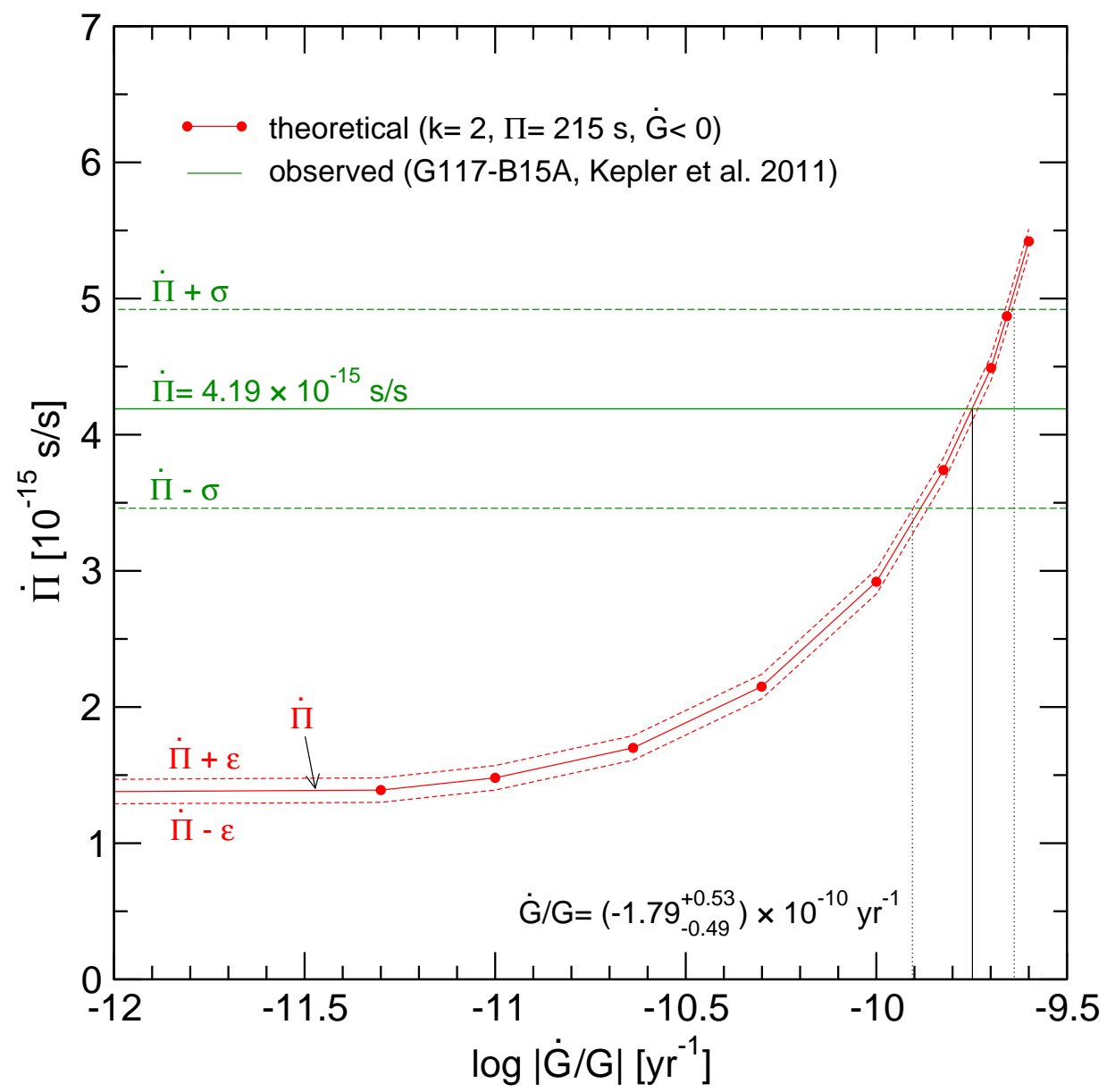

Figure 2. Rate of temporal variation of the $215 \mathrm{~s}$ period of G117-B15A as a function of the value of $\dot{G} / G$, red solid line. The effective temperature of the models is $T_{\text {eff }} \sim 11990 \mathrm{~K}$. The observational value of the rate of change of the period - horizontal solid line - along with its observed error bars — horizontal dashed lines — is also displayed. The formal theoretical errors are also shown as dashed lines.

$\varepsilon_{2} \sim 0.06 \times 10^{-15} \mathrm{~s} / \mathrm{s}[29]$. We are assuming that the uncertainty for the case in which $\dot{G} \neq 0$ is the same as that computed for the case in which $G=0$. If we consider one standard deviation from the observational value, we conclude that the secular rate of variation of the gravitational constant using G117-B15A is $\dot{G} / G=\left(-1.79_{-0.49}^{+0.53}\right) \times 10^{-10} \mathrm{yr}^{-1}$. The same analysis applied to R548 - see Fig. 3 - gives $\dot{G} / G=\left(-1.29_{-0.63}^{+0.71}\right) \times 10^{-10} \mathrm{yr}^{-1}$. These values are completely compatible each other, although currently less restrictive than those obtained using other techniques.

\section{Discussion}

We emphasize at this point that the enhanced rate of cooling of G117-B15A and R548 - which is assumed here to be entirely due to a varying $G$ - and the determination of a new upper bound for the variation of $G$ is based on the fact that our set of full DA white dwarf evolutionary models predicts that the $\ell=1, k=2$ mode $(\sim 215$ s period for G117-B15A and $\sim 213 \mathrm{~s}$ for R548) is strongly trapped in the outer $\mathrm{H}$ envelope of the 


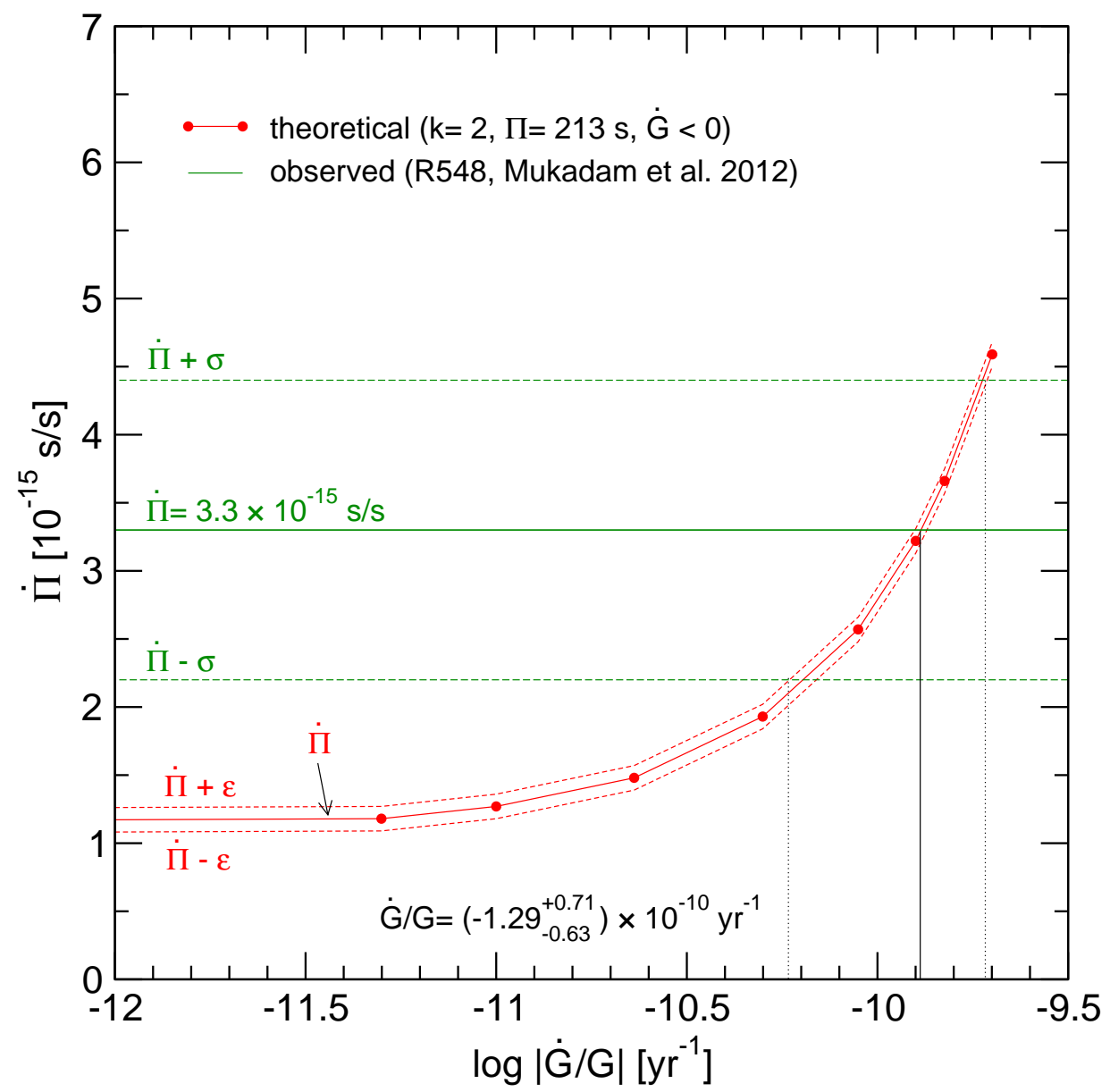

Figure 3. Same as Fig. 2 for R548. The effective temperature of the models is $T_{\text {eff }} \sim 11630 \mathrm{~K}$.

respective asteroseismological models. Had this mode not been largely trapped in the outer layers, larger values of its rate of period change would have resulted [29]. In this case, the upper bound on $\dot{G} / G$ should have been tighter. These can be estimated by considering the value of the rate of period change that the $\ell=1, k=2$ mode would have if it were nontrapped. A very simple way of doing this is to assume that the mode should have a $\dot{\Pi}$ similar to the typical value of the rate of period change of the dipole low-order non-trapped modes, that is with $\ell=1$ and $k=1,3$ and 4 for G117-B15A, and $\ell=1, k=1$ and 3 for R548. We obtain $\dot{\Pi}_{\mathrm{nt}}^{\mathrm{t}} \sim 3.9 \times 10^{-15} \mathrm{~s} / \mathrm{s}$, and $\dot{\Pi}_{\mathrm{nt}}^{\mathrm{t}} \sim 3.1 \times 10^{-15} \mathrm{~s} / \mathrm{s}$, respectively. Thus, the value of $\dot{\Pi}^{\mathrm{t}}$ for the $\ell=1, k=2$ mode of the asteroseismological model could be $\sim 2.7 \times 10^{-15} \mathrm{~s} / \mathrm{s}$ larger for G117-B15A, and $\sim 2.1 \times 10^{-15} \mathrm{~s} / \mathrm{s}$ larger for R548, if it were a non-trapped mode. Taking this into account, we obtain $|\dot{G} / G| \lesssim 10^{-11} \mathrm{yr}^{-1}$, in line with other independent upper limits, but our result is consistent with $\dot{G}=0$.

Another point that is worth discussing is the fact that we have considered only internal errors associated to the period-fit procedures that lead to the asteroseismological models for G117-B15A and R548. However, it is important to realize that substantially different asteroseismological fits (with different values for $M_{*}, T_{\text {eff }}, M_{\mathrm{H}}, \ldots$ ) are found when different sets of DA white dwarf models are employed in the analysis, being the corresponding values of $\dot{\Pi}$ for the $\ell=1, k=2$ mode quite different from those obtained here for G117-B15A and 
R548. Specifically, the spread in the theoretical value of $\dot{\Pi}$ for the $215 \mathrm{~s}$ period according to already published asteroseismological studies on G117-B15A is relatively large: $\dot{\Pi}=$ $3.7 \times 10^{-15} \mathrm{~s} / \mathrm{s}[35], \dot{\Pi}=3.9 \times 10^{-15} \mathrm{~s} / \mathrm{s}[36]$, and $\dot{\Pi}=2.98 \times 10^{-15} \mathrm{~s} / \mathrm{s}$ and $1.92 \times 10^{-15} \mathrm{~s} / \mathrm{s}$ [37]. Note, nevertheless, that all these values are smaller than the observed one $(\dot{\Pi}=4.19 \times$ $\left.10^{-15} \mathrm{~s} / \mathrm{s}\right)$. Hence, all the existing analysis indicate that G117-B15A cools faster than expected, although the spread in the value of $\dot{\Pi}$ for the $215 \mathrm{~s}$ period is rather large $\sim$ $2 \times 10^{-15} \mathrm{~s} / \mathrm{s}$. This spread can be adopted as a realistic value of the uncertainty in the theoretical rate of period change of this mode. Taking into account this uncertainty, that clearly dominates over all the errors affecting the $\dot{\Pi}^{\mathrm{t}}$, we found that $10^{-12} \lesssim|\dot{G} / G| \lesssim$ $3 \times 10^{-10} \mathrm{yr}^{-1}$, being the lower limit in very good agreement with the results obtained using other techniques. The same conclusion can be reached when R548 is considered.

\section{Summary and conclusions}

In this paper, we have used an state-of-the-art stellar evolutionary code and an up-to-date pulsational code to compute the effects of a secularly varying gravitational constant $G$ on the pulsational properties of variable white dwarfs. Specifically, we have compared the theoretical rates of period change obtained taking into account the effects of a running $G$ with the measured rates of change of the periods of two well studied pulsating white dwarfs, G117-B15A and R548. We assumed that $\dot{G} / G$ remains constant with time, and considered a rate of change of the gravitational constant in the range $5 \times 10^{-12} \mathrm{yr}^{-1} \leq|\dot{G} / G| \leq 2.5 \times 10^{-10} \mathrm{yr}^{-1}$. We found that the pulsation periods do not experience appreciable changes, but the rates of period change are strongly affected when a varying $G$ is assumed. In particular, we found that the rates of period change increase when $\dot{G} / G$ increases if $\dot{G}<0$, and the opposite holds in the case in which $\dot{G}>0$. As a result of our analysis, we have found an upper bound $|\dot{G} / G| \sim 1.8 \times 10^{-10} \mathrm{yr}^{-1}$ using the variable white dwarf G117-B15A, and $|\dot{G} / G| \sim 1.3 \times 10^{-10} \mathrm{yr}^{-1}$ using R548. These bounds are considerably weaker than those derived from Lunar Laser Ranging - $\dot{G} / G=(0.2 \pm 0.7) \times 10^{-12} \mathrm{yr}^{-1}[5]-$ and Big Bang nucleosynthesis - $-0.3 \times 10^{-12} \mathrm{yr}^{-1} \lesssim \dot{G} / G \lesssim 0.4 \times 10^{-12} \mathrm{yr}^{-1}[6,7]$ — but as stringent as the upper limits inferred from Hubble diagram of Type Ia supernovae $\dot{G} / G \lesssim 1 \times 10^{-11} \mathrm{yr}^{-1}[8,9]$. Our upper bound on the secular rate of variation of $G$ is also less restrictive than that obtained from solar helioseismology $-\dot{G} / G \lesssim 1.6 \times 10^{-11} \mathrm{yr}^{-1}$ [38]. In any case, we emphasize that while the limits reported here are not as strict as those obtained using other methods, they are independent and therefore rest on a different set of model dependent parameters.

As mentioned, our results rely on the fact that the $\ell=1, k=2$ modes are trapped in the outer $\mathrm{H}$ envelope of the asteroseismological models for G117-B15A and R548. As it is well known, mode trapping in white dwarfs is strongly sensitive to the precise shape of the chemical profiles. Since the chemical structure of white dwarfs is constructed during the evolution of their progenitor stars, a full exploration of the uncertainties affecting the white dwarf and pre-white dwarf modelling is needed to investigate if the $\ell=1, k=2$ mode in the asteroseismological models is indeed a trapped one.

In closing, we mention that the bounds obtained here using pulsating white dwarfs could be improved if the rate of period change were measured for a massive pulsating white dwarf. This is because the effects of a varying $G$ are stronger for the more massive white dwarfs [12]. Actually, it is expected that the enhancement of the cooling in a massive pulsating white 
dwarf should translate into a larger increase of its rates of period change, thus resulting in a more stringent upper limit on $\dot{G} / G$.

\section{Acknowledgments}

This research was supported by AGENCIA through the Programa de Modernización Tecnológica BID 1728/OC-AR, by PIP 112-200801-00940 grant from CONICET, by MCINN grant AYA2011-23102, by the ESF EUROCORES Program EuroGENESIS (MICINN grant EUI2009-04170), by the European Union FEDER funds, and by the AGAUR.

\section{References}

[1] P. Lorén-Aguilar, E. García-Berro, J. Isern, and Y. A. Kubyshin, Time variation of G and alpha within models with extra dimensions, Classical and Quantum Gravity 20 (Sept., 2003) 3885-3896.

[2] J. Uzan, The fundamental constants and their variation: observational and theoretical status, Reviews of Modern Physics 75 (Apr., 2003) 403-455.

[3] E. García-Berro, J. Isern, and Y. A. Kubyshin, Astronomical measurements and constraints on the variability of fundamental constants, A\&A Rev. 14 (Mar., 2007) 113-170.

[4] P. J. Mohr, B. N. Taylor, and D. B. Newell, CODATA recommended values of the fundamental physical constants: 2006, Reviews of Modern Physics 80 (Apr., 2008) 633-730.

[5] F. Hofmann, J. Müller, and L. Biskupek, Lunar laser ranging test of the Nordtvedt parameter and a possible variation in the gravitational constant, A\&A 522 (Nov., 2010) L5.

[6] C. J. Copi, A. N. Davis, and L. M. Krauss, New Nucleosynthesis Constraint on the Variation of G, Physical Review Letters 92 (Apr., 2004) 171301.

[7] C. Bambi, M. Giannotti, and F. L. Villante, Response of primordial abundances to a general modification of $G_{\mathrm{N}}$ and/or of the early universe expansion rate, Phys. Rev. D 71 (June, 2005) 123524 .

[8] E. Gaztañaga, E. García-Berro, J. Isern, E. Bravo, and I. Domínguez, Bounds on the possible evolution of the gravitational constant from cosmological type-Ia supernovae, Phys. Rev. D 65 (Jan., 2002) 023506.

[9] E. García-Berro, Y. Kubyshin, P. Lorén-Aguilar, and J. Isern, The Variation of the Gravitational Constant Inferred from the Hubble Diagram of Type ia Supernovae, International Journal of Modern Physics D 15 (2006) 1163-1174.

[10] L. G. Althaus, A. H. Córsico, J. Isern, and E. García-Berro, Evolutionary and pulsational properties of white dwarf stars, A\&A Rev. 18 (Oct., 2010) 471-566.

[11] E. García-Berro, S. Torres, L. G. Althaus, I. Renedo, P. Lorén-Aguilar, A. H. Córsico, R. D. Rohrmann, M. Salaris, and J. Isern, A white dwarf cooling age of 8 Gyr for NGC 6791 from physical separation processes, Nature 465 (May, 2010) 194-196.

[12] L. G. Althaus, A. H. Córsico, S. Torres, P. Lorén-Aguilar, J. Isern, and E. García-Berro, The evolution of white dwarfs with a varying gravitational constant, A\&A 527 (Mar., 2011) A72.

[13] E. García-Berro, M. Hernanz, J. Isern, and R. Mochkovitch, The rate of change of the gravitational constant and the cooling of white dwarfs, MNRAS 277 (Dec., 1995) 801-810.

[14] E. García-Berro, P. Lorén-Aguilar, S. Torres, L. G. Althaus, and J. Isern, An upper limit to the secular variation of the gravitational constant from white dwarf stars, J. Cosmology Astropart. Phys. 5 (May, 2011) 21. 
[15] S. O. Kepler, R. E. Nather, J. T. McGraw, and E. L. Robinson, The pulsation periods of the pulsating white dwarf G117-B15A, ApJ 254 (Mar., 1982) 676-682.

[16] S. O. Kepler, White Dwarf Stars: Pulsations and Magnetism, in Progress in Solar/Stellar Physics with Helio- and Asteroseismology (H. Shibahashi, ed.), vol. 462 of Astronomical Society of the Pacific Conference Series, p. 322, 2012.

[17] A. S. Mukadam, A. Bischoff-Kim, M. H. Montgomery, A. H. Córsico, S. O. Kepler, D. E. Winget, O. Fraser, T. S. Riecken, M. E. Kronberg, J. J. Hermes, K. I. Winget, R. E. Falcon, D. W. Chandler, J. W. Kuehne, D. J. Sullivan, D. Reaves, T. von Hippel, F. Mullally, H. Shipman, S. E. Thompson, N. M. Silvestri, and R. I. Hynes, Evolutionary Cooling of ZZ Ceti (R548), in White dwarfs (J. Krzesiński and S. Zola, eds.), Astronomical Society of the Pacific Conference Series, in press, 2012.

[18] A. D. Romero, A. H. Córsico, L. G. Althaus, S. O. Kepler, B. G. Castanheira, and M. M. Miller Bertolami, Toward ensemble asteroseismology of ZZ Ceti stars with fully evolutionary models, MNRAS 420 (Feb., 2012) 1462-1480.

[19] L. G. Althaus, A. M. Serenelli, J. A. Panei, A. H. Córsico, E. García-Berro, and C. G. Scóccola, The formation and evolution of hydrogen-deficient post-AGB white dwarfs: The emerging chemical profile and the expectations for the $P G$ 1159-DB-DQ evolutionary connection, AछA 435 (May, 2005) 631-648.

[20] L. G. Althaus, A. H. Córsico, A. Bischoff-Kim, A. D. Romero, I. Renedo, E. García-Berro, and M. M. Miller Bertolami, New Chemical Profiles for the Asteroseismology of ZZ Ceti Stars, ApJ 717 (July, 2010) 897-907, [arXiv:1005.2612].

[21] E. L. Robinson, T. M. Mailloux, E. Zhang, D. Koester, R. F. Stiening, R. C. Bless, J. W. Percival, M. J. Taylor, and G. W. van Citters, The pulsation index, effective temperature, and thickness of the hydrogen layer in the pulsating DA white dwarf G117-B15A, ApJ $\mathbf{4 3 8}$ (Jan., 1995) 908-916.

[22] D. Koester and N. F. Allard, The ZZ Ceti Instability Strip Revisited, Baltic Astronomy 9 (2000) 119-124.

[23] D. Koester and J. B. Holberg, The ZZ Ceti Instability Strip, in 12th European Workshop on White Dwarfs (J. L. Provencal, H. L. Shipman, J. MacDonald, and S. Goodchild, eds.), vol. 226 of Astronomical Society of the Pacific Conference Series, p. 299, 2001.

[24] P. Bergeron, F. Wesemael, R. Lamontagne, G. Fontaine, R. A. Saffer, and N. F. Allard, Optical and Ultraviolet Analyses of ZZ Ceti Stars and Study of the Atmospheric Convective Efficiency in DA White Dwarfs, ApJ 449 (Aug., 1995) 258.

[25] P. Bergeron, G. Fontaine, M. Billères, S. Boudreault, and E. M. Green, On the Purity of the ZZ Ceti Instability Strip: Discovery of More Pulsating DA White Dwarfs on the Basis of Optical Spectroscopy, ApJ 600 (Jan., 2004) 404-408.

[26] A. H. Córsico and L. G. Althaus, Asteroseismic inferences on $G W$ Virginis variable stars in the frame of new PG 1159 evolutionary models, A\&A 454 (Aug., 2006) 863-881.

[27] W. Unno, Y. Osaki, H. Ando, H. Saio, and H. Shibahashi, Nonradial oscillations of stars. 1989.

[28] J. P. Cox, Theory of stellar pulsation. 1980.

[29] A. H. Córsico, L. G. Althaus, M. M. Miller Bertolami, A. D. Romero, E. García-Berro, J. Isern, and S. O. Kepler, The rate of cooling of the pulsating white dwarf star G117-B15A: a new asteroseismological inference of the axion mass, MNRAS 424 (Aug., 2012) 2792-2799.

[30] A. H. Córsico, L. G. Althaus, A. D. Romero, A. S. Mukadam, E. García-Berro, J. Isern, S. O. Kepler, and M. A. Corti, An independent limit on the axion mass from the variable white dwarf star R548, J. Cosmology Astropart. Phys. 12 (Dec., 2012) 10. 
[31] P. A. Bradley, Theoretical Models for Asteroseismology of DA White Dwarf Stars, ApJ 468 (Sept., 1996) 350.

[32] J. Isern, M. Hernanz, and E. García-Berro, Axion cooling of white dwarfs, ApJ 392 (June, 1992) L23-L25.

[33] J. Isern, E. García-Berro, S. Torres, and S. Catalán, Axions and the Cooling of White Dwarf Stars, ApJ 682 (Aug., 2008) L109-L112.

[34] J. Isern, E. García-Berro, L. G. Althaus, and A. H. Córsico, Axions and the pulsation periods of variable white dwarfs revisited, A\&A 512 (Mar., 2010) A86.

[35] P. A. Bradley, Asteroseismological Constraints on the Structure of the ZZ Ceti Stars G117-B15A and R548, ApJS 116 (June, 1998) 307.

[36] A. H. Córsico, O. G. Benvenuto, L. G. Althaus, J. Isern, and E. García-Berro, The potential of the variable DA white dwarf G117-B15A as a tool for fundamental physics, New A 6 (June, 2001) 197-213, [astro-ph/].

[37] A. Bischoff-Kim, M. H. Montgomery, and D. E. Winget, Strong Limits on the DFSZ Axion Mass with G117-B15A, ApJ 675 (Mar., 2008) 1512-1517, [arXiv:0711.2041].

[38] D. Guenther, L. Krauss, and P. Demarque, Testing the constancy of the gravitational constant using helioseismology, Astrophys. J. 498 (1998) 871-876. 